\title{
Polymorphisms in detoxification genes increase HMTC risk and match tumour phenotype
}

Genes that encode enzymes responsible for the detoxification of environmental carcinogens are associated not only with the risk of development of hereditary medullary thyroid carcinoma (HMTC) but also with the clinical characteristics of these tumours, say researchers from Brazil.

A team lead by senior investigator Laura S. Ward (University of Campinas) had previously demonstrated that detoxification genes such as GSTP1, NAT2 and TP53 are important determinants of the susceptibility to differentiated thyroid cancer. Moreover, the researchers had implicated these genes in the development of sporadic medullary thyroid carcinomas and autoimmune thyroid conditions, such as Graves disease.

\section{We may now be able to}

explain why ... the disease

develops earlier, more

\section{aggressively or not at all 77}

"Detoxification genes modulate the effect of environmental and endogenous factors that deeply impact our organism in various ways," explains Ward. "Hence, they could also influence genetically determined diseases such as HMTC." The researchers tested this hypothesis by genotyping 132 patients with HMTC,
88 first-degree relatives of these patients and 575 control individuals for polymorphisms in the detoxification genes CYP1A2*F, CYP1A1m1, GSTP1, NAT2 and TP53. Their goal was to find specific profiles that could help identify individuals at risk and thus determine specific management and/or preventive measures.

The results showed that the inheritance of a specific profile of detoxification genes is associated with the risk of HMTC as well as with phenotypic tumour characteristics. "This finding adds a totally new perspective to the traditional understanding of hereditary tumours (and perhaps other complex diseases as well)," says Ward. "We may now be able to explain why patients, despite bearing the exact same genes that determine a hereditary condition, such as the RET oncogene, present with different clinical phenotypes; in other words, why in some patients the disease develops earlier, more aggressively or not at all."

Furthermore, Ward and co-workers identified individuals at high risk of developing HMTC among the relatives of patients with these tumours. Given that these individuals might develop tumours associated with specific carcinogens, Ward suggests that they receive special attention: "for instance, these patients should be advised not to smoke or to avoid exposure to pollutants".

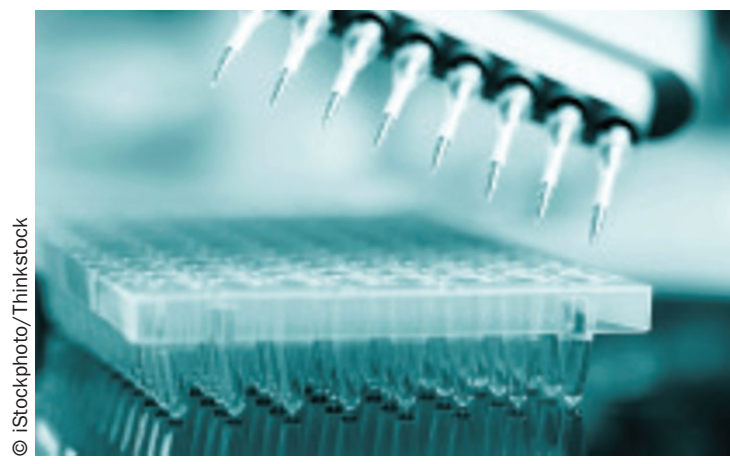

The investigators aim to analyse the interaction of other previously described polymorphisms of the RET oncogene with a large profile of detoxification genes, for which they require a larger population of patients with HMTC than the one investigated in the current study.

"I believe that in the near future we will be able to genotype and identify groups of risk for specific chronic diseases, including cancer, autoimmune and complex conditions," comments Ward. "We will be able to provide precocious orientation on lifestyle for each individual to prolong and improve quality of life."

Linda Koch

Original article Barbieri, R. et al. Genes of detoxification are important modulators of hereditary medullary thyroid carcinoma risk. Clin. Endocrinol. (Oxf.) doi:10.1111/ cen.12136 